\title{
因HAD
}

DOI: http://doi.org/10.22585/hospdomic.v3i1.61

\section{Hacia una normalización de la representación de flujogramas en el ámbito hospitalario}

\section{Towards a normalization on the representation of workflows in the hospital field}

Mateo Ramos Merino', Juan M. Santos Gago', Luis M. Álvarez Sabucedo'

1. Escola de Enxeñaría de Telecomunicación de la Universidad de Vigo, Vigo, España.

Correspondencia/Correspondence

Luis M. Álvarez Sabucedo

Isabucedo@gist.uvigo.es

Recibido/Received

27.11 .2018

Aceptado/Accepted

29.12 .2018
Conflicto de Intereses/Competing interest No existe ningún conflicto de interés en el presente estudio

Agradecimientos/Acknowledgments

Este trabajo ha sido parcialmente financiado con una ayuda del Instituto de Salud Carlos III de Madrid, España, mediante el proyecto PI16/00788 (Control de la calidad y la trazabilidad de los medicamentos biopeligrosos (MBP) a través del Hospital a Domicilio mediante modelos Big Data aplicados al análisis de riesgos.) 


\section{RESUMEN}

En este artículo se presenta una propuesta de normalización para la representación de protocolos hospitalarios a través de diagramas de flujo. En el ámbito clínico se desarrollan procesos de gran complejidad que, si no se gestionan adecuadamente, podrían dar lugar a consecuencias fatales para los pacientes o el personal sanitario implicado. Utilizar una notación clara que formalice las tareas que deben realizarse es un aspecto fundamental para mantener los riesgos controlados. En la práctica, el lenguaje de flujogramas más utilizado desde hace años debido a su sencillez y versatilidad es el definido por la norma ISO 5807:1985. No obstante, este lenguaje resulta insuficientemente expresivo para representar todos los aspectos importantes de un protocolo sanitario. Durante los últimos años este tipo de protocolos han aumentado su complejidad con el fin de dar un paso hacia adelante en el control de aspectos críticos que influyen en la seguridad de los medicamentos, pacientes y personal sanitario. En esta línea, una práctica muy común ha sido la implantación de la metodología APPCC (Análisis de Peligros y Puntos Críticos de Control). Durante el modelado de estos protocolos (dado que la notación de flujogramas tradicional no es suficientemente expresiva) cada centro hospitalario ha adoptado una propuesta propia que complementa los elementos incluidos en la norma ISO (International Organization for Standardization). Con esta práctica se obliga a que el personal tenga que familiarizarse con la notación definida ad-hoc para ese centro que, además de no estar estandarizada, cae en ocasiones en definiciones ambiguas y poco formales. En la propuesta de normalización presentada en este artículo se plantean nuevos estereotipos gráficos fácilmente integrables en la notación ISO y diseñados para representar los aspectos críticos de la metodología APPCC. Para la definición de estos nuevos elementos se han priorizado una serie de requisitos que tienen en cuenta tanto los aspectos relativos a las herramientas software ya existentes como la simplicidad para los usuarios finales en la práctica diaria. Al mismo tiempo se tratan de evitar ambigüedades asegurando que los elementos introducidos no interfieren con la notación base contenida en la norma ISO original. En definitiva, esta propuesta de normalización consigue enriquecer el lenguaje ISO5807:1985 con mecanismos de expresión suficientes para representar la información básica relacionada con la seguridad de los protocolos reduciendo las ambigüedades y facilitando una notación estandarizada.

Palabras clave: Procesamiento Automatizado de Datos; Técnicas de Apoyo para la Decisión; Sistemas de Apoyo a Decisiones Clínicas; Toma de Decisiones Asistida por Computador; Árboles de Decisión; Estándares de Referencia; Análisis de Sistemas.

\section{ABSTRACT}

This article presents a proposal of standardization for the representation of hospital protocols using flowcharts. In the clinical field, highly complex processes are typically developed. If these processes are not appropriately managed, fatal consequences for patients and the healthcare personnel could appear. Using a clear notation for modelling the tasks to be performed is mandatory to keep the risks under control. In practice, the most widely used flowchart language for years (due to its simplicity and versatility) is defined in ISO 5807:1985 standard. However, this language is insufficiently expressive to represent all the essential aspects of a health protocol. In recent years, this type of protocols has become increasingly sophisticated in order to improve the control of critical aspects that influence the safety of medicines, patients and practitioners. In this way, a widespread practice has been the implementation of the HACCP methodology for hazard analysis and control. During the modelling of these protocols (and because of the expressivity problems of traditional flowchart notation), each hospital has adopted its proposal that complements the elements included in the ISO (International Organization for Standardization) standard. This practice forces practitioners to become familiar with the ad-hoc defined notation for that center. In addition, these adaptations usually fall into ambiguous and non-formal definitions. In the standardization proposal presented in this article, new graphic stereotypes are introduced. These can be easily integrated into the ISO notation and are designed to represent the critical aspects of the HACCP (Hazard Analysis and Critical Control Points) plan. 
For the definition of these new elements, a series of requirements have been prioritized. First, it is taken into account the tools that are commonly used and the simplicity for final users. Moreover, it is mandatory to avoid ambiguities assuring that the introduced elements do not interfere with the base notation contained in the original ISO standard. This standardization proposal enriches the ISO5807:1985 language with expression mechanisms to represent essential information related to protocol security by reducing ambiguities and proposing a standardized notation.

Keywords: Automatic Data Processing; Decision Support Techniques; Decision Support Systems, Clinical; Decision Making, Computer-Assisted; Decision Trees; Reference Standards; Systems Analysis.

\section{INTRODUCCIÓN}

Los centros hospitalarios son entidades de gran complejidad. Los errores en los procesos propios de estos centros pueden dar lugar a consecuencias fatales tanto para los pacientes como para el personal que allí desempeña su trabajo. Desde una perspectiva formal, entendemos como «proceso" a un conjunto de actividades interrelacionadas que se llevan a cabo de forma ordenada por parte de un grupo de actores para alcanzar un fin predefinido. En el ámbito hospitalario se pueden identificar procesos primarios (e.g. procedimientos médicos o quirúrgicos), procesos de apoyo (e.g. mantenimiento o limpieza) y procesos de gestión. Una ejecución correcta y segura de estos procesos hospitalarios es fundamental para garantizar un servicio eficaz y de calidad.

Una práctica común para minimizar los errores y las situaciones no deseadas derivadas de una realización incorrecta de un protocolo es normalizarlo. Esto se consigue definiendo un protocolo consensuado que describa a priori cómo deben proceder los actores involucrados en cada momento para alcanzar el fin deseado. Estos protocolos se plasman en documentos que, dependiendo del ámbito de actuación, pueden tomar diferentes formas: fichas de protocolos de trabajo, protocolos asistenciales, mapas de protocolos, guías de práctica clínica, vías clínicas, etc. Los protocolos normalizados permiten, por un lado, sistematizar los procesos, minimizando de este modo los fallos de ejecución, y, por otro lado, reducir el impacto y las consecuencias de posibles errores e imprevistos, cuando incluyen guías de actuación ante este tipo de situaciones. También es común incluir en los protocolos planes de inspección, facilitando de esta manera la auditoría de procesos y el control de la calidad.

Un protocolo, es decir, un proceso que ha sido normalizado, se plasma en un documento o conjunto de documentos que va a ser utilizado por los responsables de su implantación, algunos de los actores que participan en las actividades involucradas y, potencialmente, los encargados de supervisarlo y auditarlo. Huelga decir que estos documentos debieran ser de fácil interpretación y asimilación por parte de estos "lectores". Típicamente los documentos incluyen textos convenientemente estructurados para facilitar su interpretación. Es muy común, también, el uso de elementos gráficos sencillos que representan visualmente el flujo de las actividades involucradas en el proceso. Estos flujogramas son fundamentales en la documentación de protocolos, tanto más cuanto más complejo son, pues condensan mucha información en poco espacio y facilitan una rápida y eficaz comprensión y comprobación de las actividades que conforman un protocolo y el orden en que deben ejecutarse. Sin embargo, a pesar de su importancia, estos elementos gráficos son utilizados de manera informal y de modo heterogéneo en las diferentes notaciones que normalizan los protocolos hospitalarios.

Con el fin de contribuir a reducir este problema, hemos establecido una serie de recomendaciones para la representación de flujogramas en el ámbito hospitalario. Este modelo está basado 
en el uso de una extensión ad-hoc sobre la norma ISO 5807:1985 (3), un lenguaje muy popular de representación de flujogramas, que incluye elementos específicos para representar de manera simple protocolos que incluyen planes de inspección de manera explícita. En este artículo se presenta brevemente esta propuesta de notación. El artículo se organiza del siguiente modo: la sección 2 describe el panorama actual relativo al uso de flujogramas en la documentación de los protocolos hospitalarios y pone de relieve algunos de los problemas detectados; la sección 3 expone la notación propuesta, orientada a paliar parcialmente estos problemas; y, por último, la sección 4 resume y concluye el artículo.

\section{ESTADO DEL ARTE}

Un examen de múltiples protocolos sanitarios y hospitalarios de diferente índole ha permitido poner de relieve un uso relativamente frecuente de los flujogramas como elemento de representación visual de los procesos. Estos elementos gráficos claramente permiten enriquecer y mejorar la comprensión de los mismos. El examen de los protocolos ha puesto de manifiesto, también, la gran variedad de soluciones utilizadas por los diseñadores para plasmar estos gráficos. En muchas ocasiones, los flujogramas son representaciones sencillas de la secuenciación de las actividades implicadas en los procesos utilizando rectángulos para identificar actividades y conectores en forma de flechas para definir el paso de una actividad a otra. En este sentido, la figura 1 muestra un ejemplo típico de estos flujogramas, que si bien son autoexplicativos, no parecen seguir una notación estandarizada de representación de flujogramas.

Figura 1. Flujograma disponible en la «Guía de Práctica Clínica sobre Terapia Intravenosa con Dispositivos no Permanentes en Adultos» (1)

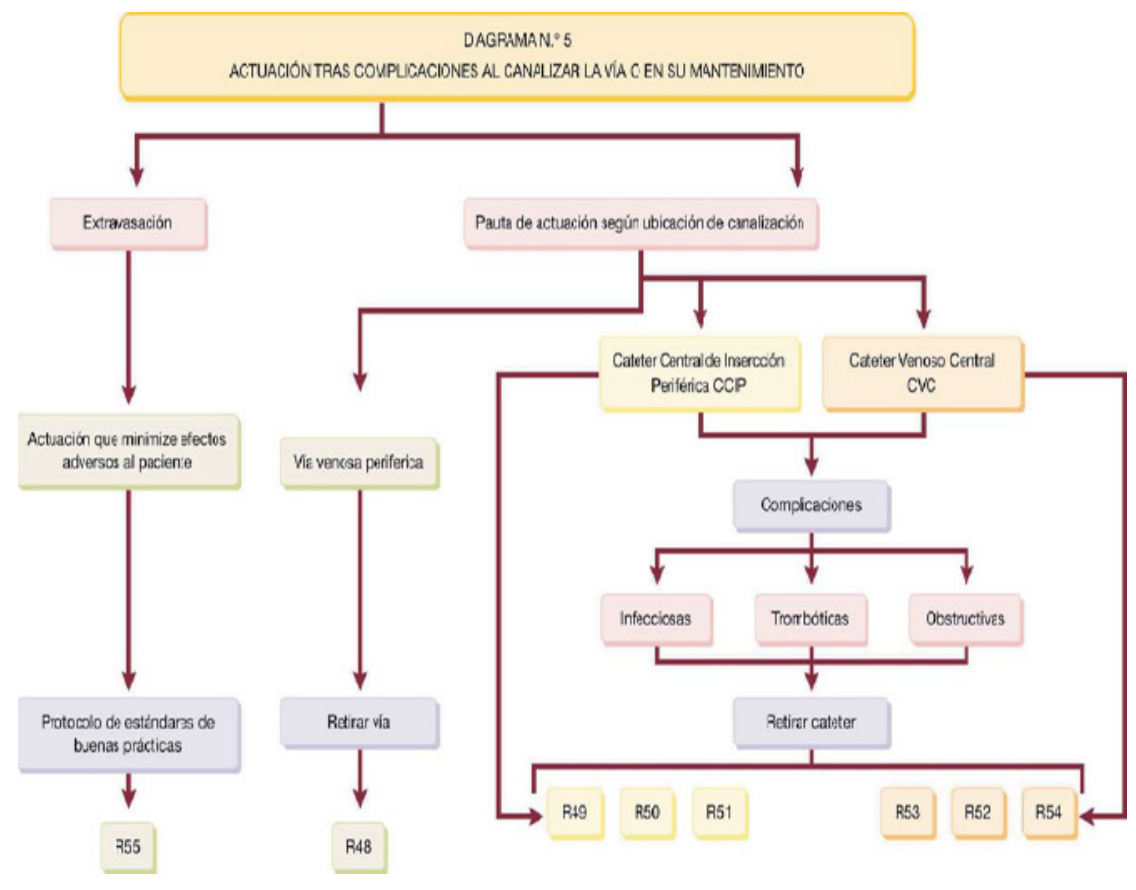


Cabe mencionar que la interpretación de este flujograma es sencilla, sobre todo para una persona con dominio en el ámbito de actuación, aunque, desde una perspectiva formal, es destacable la ausencia de elementos gráficos para la representación de "decisiones" o el uso de colores sin una semántica predefinida. La figura 2 muestra un flujograma de características similares al anterior, si bien en este caso sí se utilizan elementos decisores (hexágonos).

Figura 2. Flujograma incluido en la «Guía de Práctica Clínica sobre lactancia materna» (2)

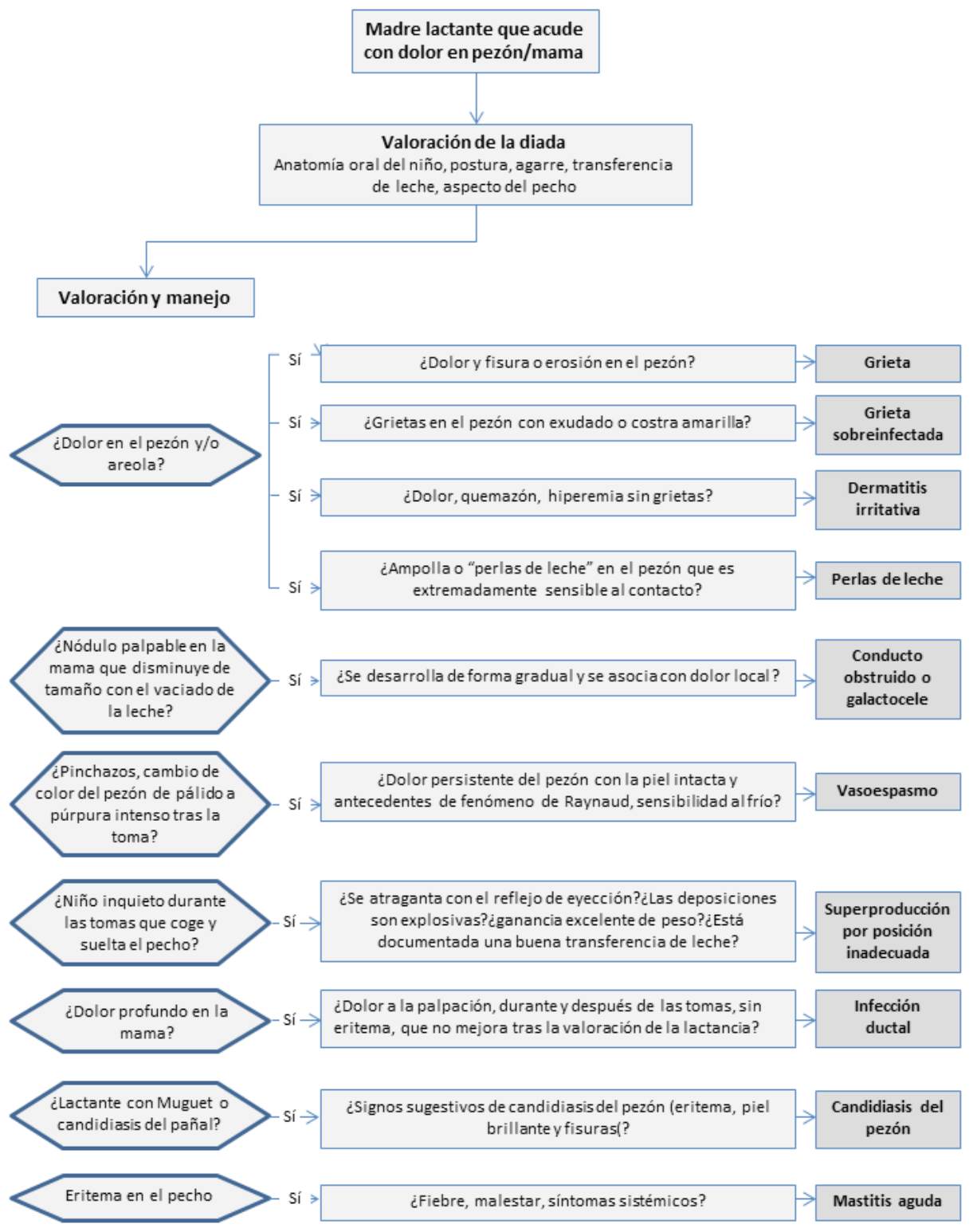


También es posible encontrar con bastante frecuencia flujogramas cuya representación es fiel o muy cercana a la norma ISO 5807:1985 (3). La figura 3 es un ejemplo de esto. En este caso los decisores son rombos, reservándose los hexágonos para representar actividades de preparación o inicialización del proceso, tal como establece la notación ISO 5807:1985. Es destacable en este flujograma que los colores asignados a los diferentes elementos tienen una semántica predefinida (por ejemplo, el color rojo intenso está asignado a los elementos que tratan algún tipo de riesgo grave). Sin embargo, en este diagrama el hexágono se utiliza, además del rombo, como decisor. Lo cual, si bien no se puede decir que contradice la norma ISO 5807:1985, sí se puede afirmar que está poco alineado con su semántica y el uso común del elemento gráfico hexágono.

Figura 3. Flujograma incluido en la «Guía de Práctica Clínica sobre el Manejo de la Depresión en el Adulto» (4)

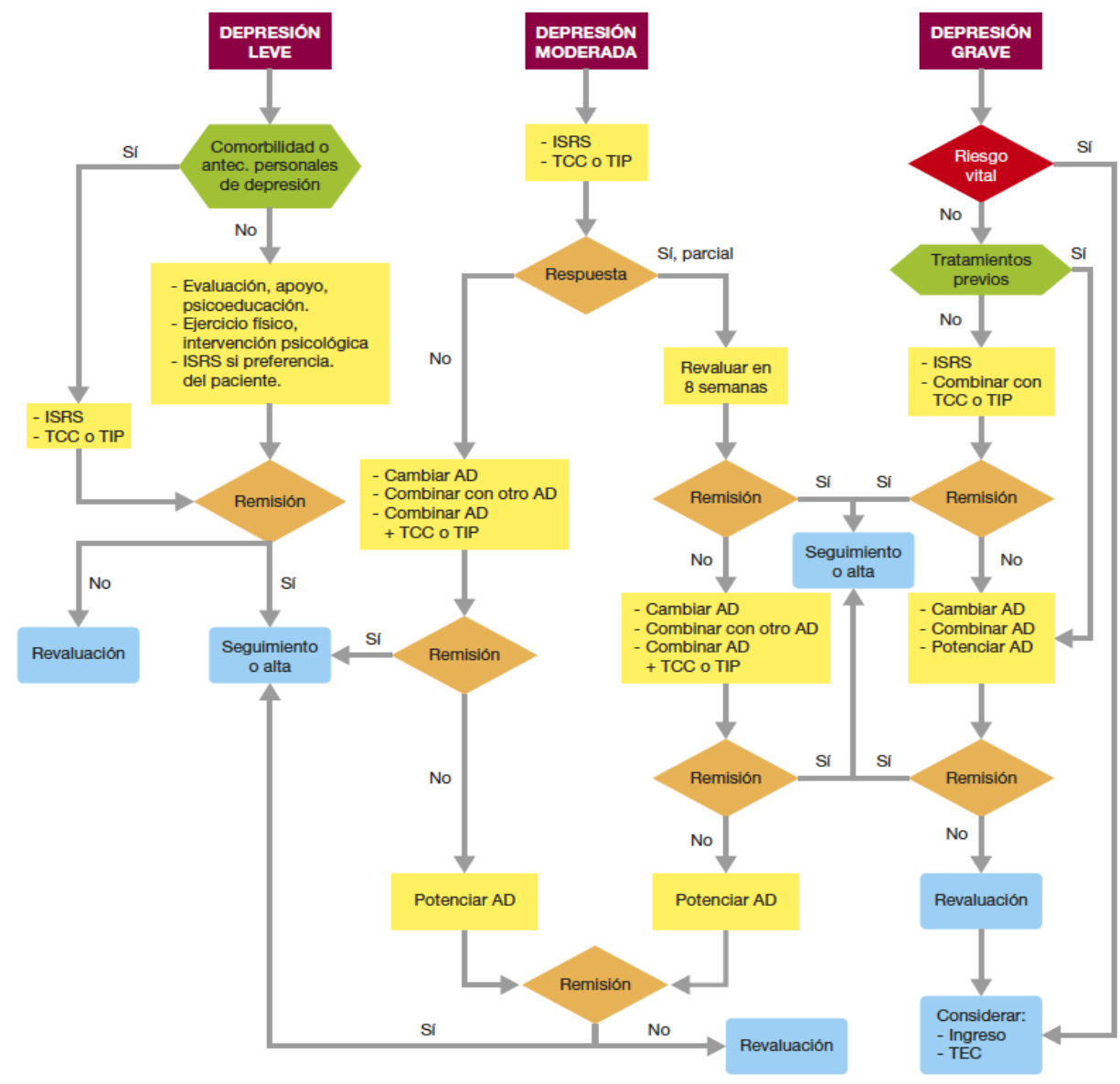

El uso de una notación estandarizada como la norma ISO 5807:1985 para modelar este tipo de diagramas es aconsejable, pues de esta manera se uniformiza la manera de representar los flujogramas. La notación ISO 5807:1985 es un lenguaje gráfico sencillo, con pocos elementos, de uso extendido y fácil de entender. Su empleo redunda en una mayor legibilidad y en una más eficiente interpretación de los flujogramas. 
En cualquier caso, la norma ISO no está exenta de inconvenientes:

- En primer lugar, es una norma muy laxa en su semántica, lo que da lugar a diferentes modos de representación de una misma secuenciación de actividades por parte de diferentes diseñadores, llegando a producirse, con frecuencia, ambigüedades.

- En segundo lugar, posee un potencial expresivo limitado, lo que supone un problema tanto para describir información de contexto como para representar de manera explícita comportamientos de secuenciado complejo (e.g. actividades de realización condicionada).

Existen notaciones gráficas con mucho mayor potencial expresivo, como UML (Unified Modeling Language) (5), BPMN (Business Process Model and Notation) (6), YAWL (Yet Another Workflow Language) (7) o incluso las Redes de Petri (8). Algunas de estas notaciones han sido utilizadas de manera alternativa a la norma ISO 5807:1985 para la representación formal de flujogramas complejos en protocolos sanitarios y hospitalarios. En particular, extensiones de la notación BPMN, un lenguaje para modelar gráficamente procesos muy popular en el ámbito de la industria, han sido propuestas en diversos trabajos. Por ejemplo, en los trabajos de Braun et al. $(9,10)$, se presenta una extensión de BPMN para el ámbito de los procesos clínicos que permite diferenciar gráficamente los distintos tipos de tareas (diagnósticas, terapia, soporte, etc.). Otro ejemplo es propuesto por Strasser et al. (11), donde se propone una nueva manera de representar los procesos de cuidados clínicos con el objetivo de identificar las tareas que tienen un alto coste. Los propios autores de este artículo han contribuido en el área proponiendo la extensión BPMNE2 (BPMN Enhanced Expressiveness) (12-14), que aprovecha todas las ventajas del lenguaje BPMN y añade nuevos estereotipos gráficos que permiten, entre otras cosas, distinguir actividades de inspección (para el seguimiento y control de calidad de los procesos) de manera clara y explícita, facilitando su interpretación no sólo por personas sino también por agentes software.

Estas propuestas alternativas solucionan los problemas de carencia de una semántica formal y de expresividad reducida de la norma ISO 5807:1985. No obstante, una consecuencia inevitable de su alta expresividad es la incorporación de nuevos elementos gráficos dotados de una semántica muy precisa. Esto, unido a la falta de herramientas maduras para su uso, conlleva una curva de aprendizaje muy tendida. Por lo tanto, su coste, tanto en tiempo como en recursos para su utilización puede resultar desalentadora en algunos contextos.

\section{PROPUESTA}

Con el fin de paliar parcialmente los problemas identificados en la sección anterior, se ha decidido definir una extensión de la norma ISO 5807:1985 que contemple la posibilidad de representar de manera clara planes de actuación que, al mismo tiempo, faciliten la auditoría de los procedimientos. Actualmente, hasta donde los autores conocen, no hay ninguna norma basada en ISO 5807:1985 que explicite cómo incluir en los flujogramas actividades de supervisión y control de calidad. Los diseñadores de protocolos suelen utilizar anotaciones personales para identificar qué tareas están relacionadas con la monitorización de los procesos (ya sea mediante el uso de elementos propios de la norma ISO 5807:1985 para la inclusión de comentarios, el resaltado de actividades mediante colores específicos o, incluso, escribiendo a mano notas en la documentación impresa). Esto provoca que los diagramas de procesos hospitalarios de distintos diseñadores sean incompatibles entre sí, al no seguir una notación normalizada.

Con respecto al diseño de planes de inspección y de control, cada vez se usa con mayor profusión la metodología APPCC (Análisis de Peligros y Puntos Críticos de Control) (15). Haciendo uso 
de esta metodología, a la par que se diseña un protocolo, se realiza un estudio en el que se analizan los peligros potenciales que pueden estar asociados a cada actividad. Este análisis permite identificar Puntos de Control (PC), que se asocian con aquellas actividades que tienen relación con la seguridad del proceso. Estos PC cobran especial importancia a la hora de trazar e inspeccionar los procesos. Dentro de estos, se identifican también Puntos Críticos de Control (PCC). Siendo estos últimos, aquellos en los que la probabilidad de que surja un problema y su potencial peligrosidad, obliga a tratarlos de un modo crítico. En los PCC es muy importante garantizar que se cumplan las restricciones y condiciones preestablecidas, de modo que no se superen determinados límites críticos, pues lo contrario podría suponer problemas graves de salud para el paciente o para el personal sanitario. Un ejemplo de un PCC podría ser el control de la fecha de caducidad de un medicamento peligroso o la comprobación de la integridad de un filtro.

En este trabajo se propone una extensión de la notación ISO 5807:1985 que permite dar soporte básico a los elementos más importantes de un plan APPCC. De este modo, se facilita el traslado de la información más relevante contenida en el plan hacia el flujograma. En la propuesta se ha priorizado un diseño que posibilite una transición suave desde el modo habitual en la actualidad de representar flujogramas, aunque ello pueda limitar la potencia expresiva de la nueva notación. En particular, para la definición de los elementos gráficos que conforman la extensión se tuvo en consideración: i) que las herramientas de uso común en la actualidad para la edición de flujogramas (e.g. Microsoft Visio, ...) incluyesen los elementos incorporados, ii) que estos elementos incorporados fuesen fácilmente dibujados a mano, iii) que los nuevos elementos no formen parte del conjuntos de elementos de la norma ISO original ni se correspondan con elementos utilizados con frecuencia de manera oficiosa, iv) y que fuesen reconocibles fácilmente cuando se imprimen tanto en color como en escala de grises.

A continuación se detallan los nuevos elementos gráficos propuestos:

Punto de Control (PC), permite identificar aquella tarea en la que se ha establecido un Punto de Control que no es Crítico y establece, en el diagrama de flujo, la condición que fija la conformidad con dicho PC. En el caso en el que la condición no sea satisfecha es posible definir un nuevo flujo alternativo que parte del PC y que contendrá aquellas actividades necesarias para la subsanación de los problemas encontrados. El elemento gráfico correspondiente a una actividad identificada como PC se muestra en la Figura 4. Se incluye una propuesta para flujogramas que sean representados en color y otra para flujogramas en escala de grises.

Figura 4. Estereotipo gráfico correspondiente a un Punto de Control Crítico (PCC)
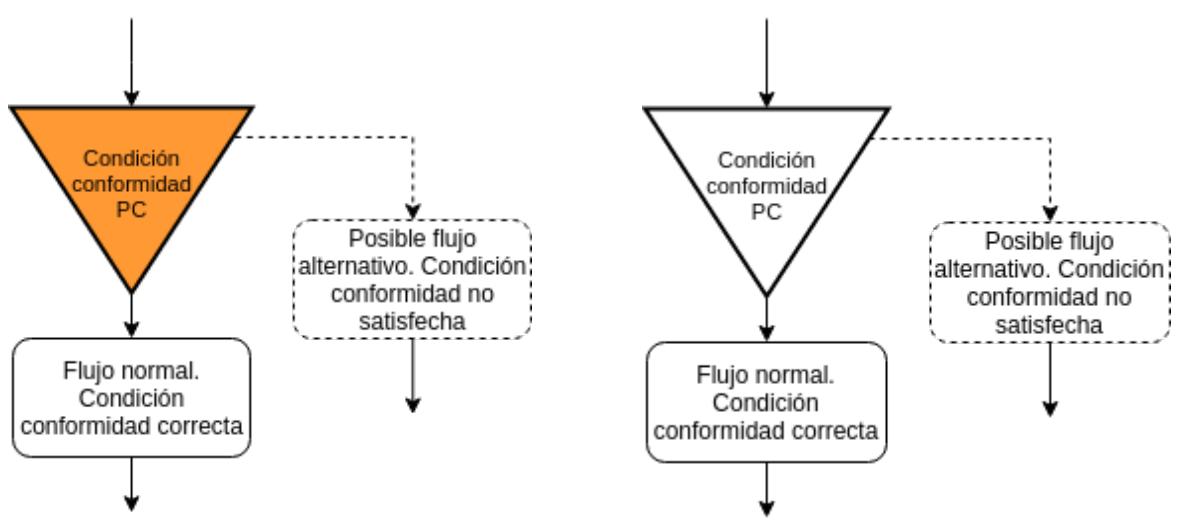
Se utiliza para identificar aquella actividad en la que se ha establecido un Punto Crítico de Control y establece las condiciones que fijan la conformidad con dicho PCC. Cuando el límite crítico que establece el PCC sea excedido y, por tanto, la conformidad no sea satisfecha se definirá un nuevo flujo alternativo que parte del PCC y que contendrá aquellas actividades necesarias para el correcto tratamiento del producto afectado. En la Figura 5 se incluye una propuesta de elemento gráfico para flujogramas que sean representados en color y otra para flujogramas en escala de grises.

Figura 5. Estereotipo gráfico correspondiente a un Punto de Registro de Información (PRI)
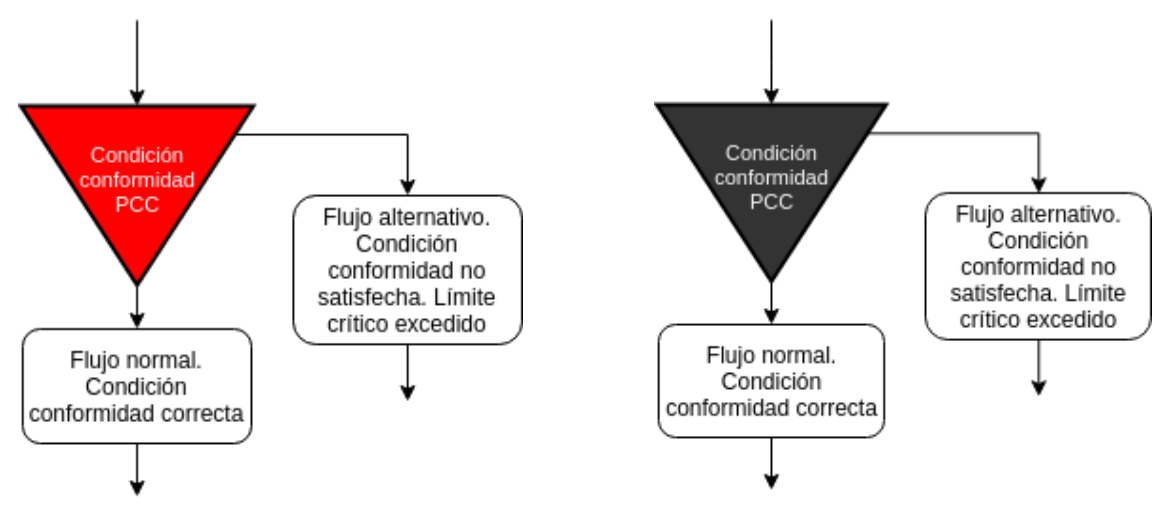

A lo largo de la ejecución de un proceso es común que se realicen mediciones de ciertas características contextuales o de determinados parámetros asociados a los productos que se manejan en determinadas actividades. Esto permite recabar información para conocer el estado del proceso y comprobar, típicamente en los PC y PCC, si se cumplen las restricciones de seguridad. El estereotipo mostrado en la Figura 6 se corresponde con la propuesta de elemento gráfico orientado a identificar de manera aquella actividad en la que se lleva a cabo el registro de información y cuáles son los objetos de la monitorización (i.e. variables que se medirán). Dentro del estereotipo se permite la descripción en formato libre de las mediciones previstas.

Figura 6. Estereotipo gráfico correspondiente a un Punto de Registro de Información

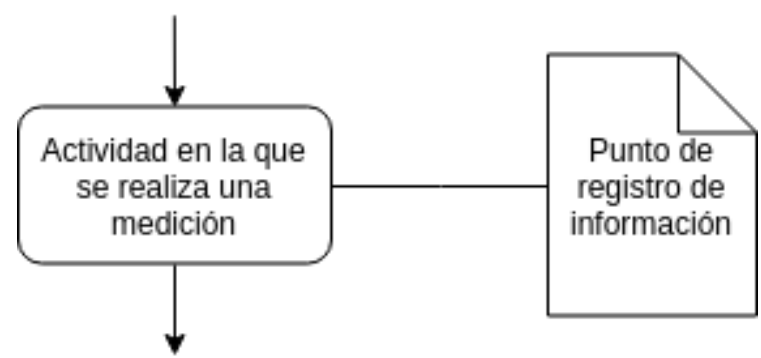


La figura 7 presenta un ejemplo sencillo de flujograma que incluye los elementos identificados. En el ejemplo se muestra un fragmento de un proceso de administración de un medicamento. En el flujograma se pueden localizar dos Puntos de Registro, uno para anotar la caducidad y el paciente receptor y otro para medir la temperatura; un Punto de Control, utilizado para comprobar que se cumplen las restricciones de temperatura; y un Punto de Control Crítico que contiene una condición que de no cumplirse supone la retirada del medicamento.

Figura 7. Ejemplo de flujograma que utiliza los nuevos elementos gráficos

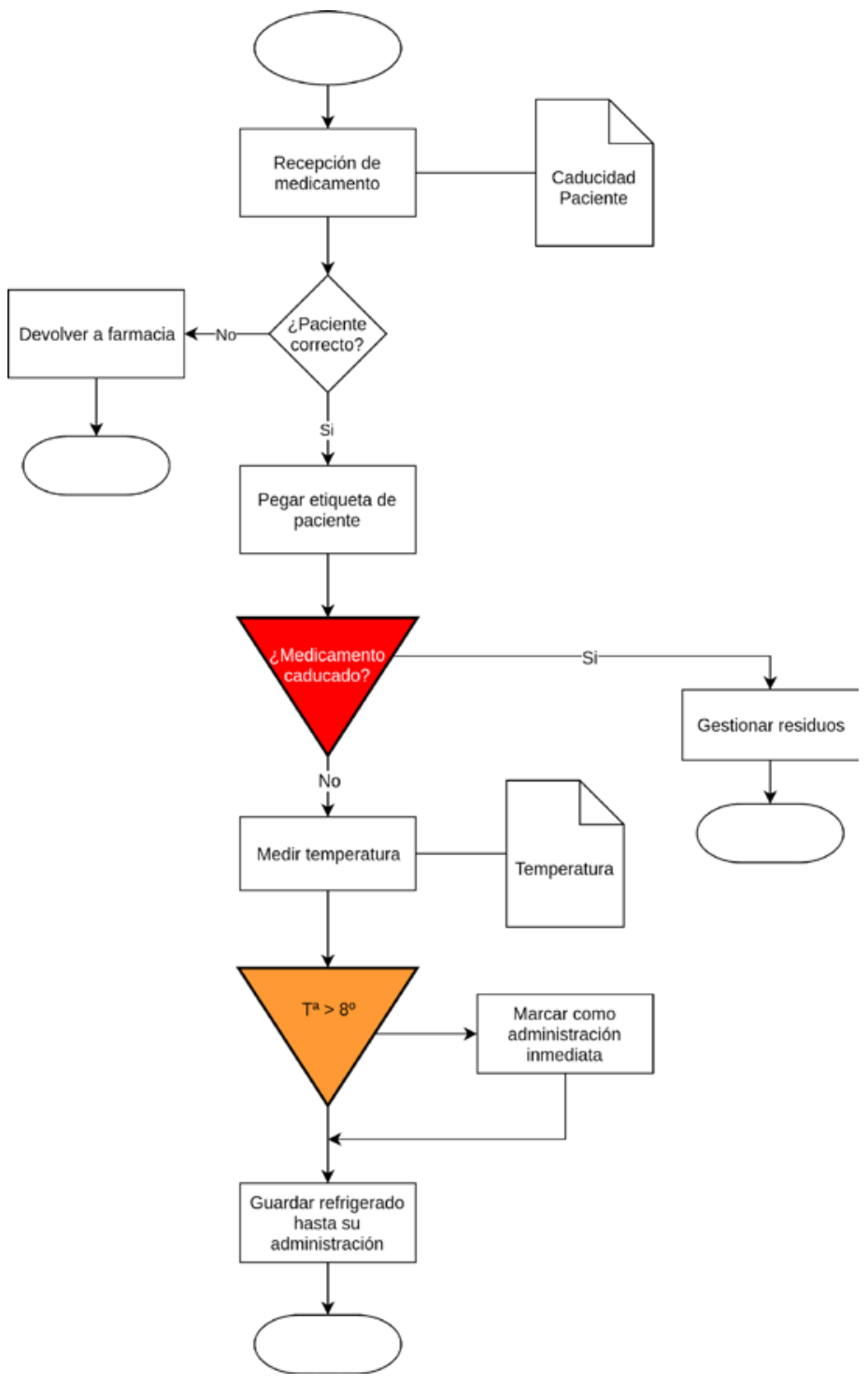




\section{CONCLUSIONES Y LÍNEAS FUTURAS}

En este artículo se ha presentado una propuesta de notación para representar flujogramas en procesos hospitalarios que extiende la norma ISO 5807:1985. El objetivo subyacente ha sido el de dotar a la norma ISO 5807:1985 de mecanismos expresivos suficientes para representar explícitamente y con claridad la información más relevante contenida en un plan APPCC. La utilización de la notación propuesta supone un avance potencial en la normalización de los procesos hospitalarios. Con una pequeña ampliación de la ISO 5807:1985, la notación utilizada comúnmente por los diseñadores de protocolos hospitalarios y un coste de introducción mínimo, se pueden conseguir grandes ventajas derivadas de la estandarización del lenguaje gráfico. El fin último ha sido mantener la notación tradicional y dotarla de unos nuevos elementos que solucionan los aspectos más importantes de la problemática expuesta en la sección 2. La Tabla 1 condensa una comparativa entre la notación ISO 5807:1985 y su extensión. El uso de ISO 5807:1985 como base permite reducir la curva de aprendizaje de los auditores y personal clínico (ya que los fundamentos del lenguaje resultan conocidos), solo siendo necesario familiarizarse con los nuevos elementos.

Tabla 1. Comparación entre la notación ISO 5807:1985 y su extensión

\begin{tabular}{|l|l|l|}
\hline ISO & ISO+APPCC \\
\hline $\begin{array}{l}\text { Complejidad de la } \\
\text { notación }\end{array}$ & $\begin{array}{l}\text { Notación gráfica muy sencilla, } \\
\text { intuitiva y fácil de aprender }\end{array}$ & $\begin{array}{l}\text { Al incorporar más elementos aumenta } \\
\text { ligeramente la complejidad de la } \\
\text { notación }\end{array}$ \\
\hline $\begin{array}{l}\text { Presencia de } \\
\text { ambigüedades }\end{array}$ & $\begin{array}{l}\text { Presenta ambigüedades con } \\
\text { frecuencia. No hay una manera } \\
\text { normalizada de representar la } \\
\text { información del plan APPCC }\end{array}$ & $\begin{array}{l}\text { Reduce ambigüedades en los } \\
\text { flujogramas que incluyen plan de } \\
\text { inspección. Existen estereotipos gráficos } \\
\text { orientados a representar los elementos } \\
\text { clave de un plan APPCC }\end{array}$ \\
\hline $\begin{array}{l}\text { Grado de conocimiento } \\
\text { visualizando la notación }\end{array}$ & $\begin{array}{l}\text { Se podría llegar a intuir la situación } \\
\text { de los PC y PCC pero presenta } \\
\text { ambigüedades }\end{array}$ & $\begin{array}{l}\text { Vuelca la información más importante al } \\
\text { diagrama gráfico. Con esto se permite } \\
\text { un mayor entendimiento del protocolo } \\
\text { tan solo consultando el diagrama. Con } \\
\text { un golpe de vista se puede conocer } \\
\text { información completa acerca de los } \\
\text { elementos más importantes del plan } \\
\text { APPCC }\end{array}$ \\
\hline
\end{tabular}

Además, los autores han observado también un curioso efecto al introducir la notación en los ámbitos finales de uso. Muchos trabajadores del entorno, al tener que utilizar los modelos propuestos, se hicieron mucho más conscientes de la existencia de los PCC ya que tenían estereotipos específicos para su inclusión en el modelo final. Y lo que resulta más interesante aún, muchos empezaron a pensar en términos de estos ya que su impacto en el modelo global era mucho más evidente. Se observó, nuevamente, un caso más de la vieja máxima de que el lenguaje crea realidad.

Este trabajo forma parte de un proyecto de mayor envergadura cuyos resultados parciales han sido recogidos en diversos artículos. En el trabajo de Bernabeu-Martínez et al. (16), se presenta una revisión sistemática de las guías de prácticas clínicas relacionadas con el ámbito de los medica- 
mentos peligrosos (MP). Tras este estudio se ha puesto de manifiesto que no existen en la actualidad protocolos globalmente armonizados para el manejo y prevención de riesgos de exposición a MP ni sistemas informáticos adecuados que permitan gestionar la calidad, la trazabilidad y la minimización de los riesgos asociados a estos medicamentos. Con el objetivo de mejorar la expresividad de los protocolos clínicos, los autores han realizado la propuesta de la notación BPMNE2 (12), basada en el lenguaje BPMN y que ha sido validada en el ámbito hospitalario (13). Esta notación es más compleja y completa que la propuesta en el presente artículo y, por lo tanto, será apropiada en el caso en el que el contexto de aplicación pueda soportar un coste de cambio mayor con una curva de aprendizaje más pronunciada. Un punto muy importante es que esta notación proporciona los mecanismos necesarios para que sea entendible por máquinas, permitiendo el control de los procesos a través de la trazabilidad informatizada. Esto abre la puerta a un amplio abanico de posibilidades de implantación de diversas técnicas y algoritmos de análisis basados en data mining (17) o big-data (18). En la actualidad se está trabajando para obtener beneficio de estas tecnologías aplicándolas con el objetivo de mejorar las tareas de auditoría y control de los procesos.

\section{BIBLIOGRAFÍA}

1. Grupo de trabajo de la Guía de Práctica Clínica sobre Terapia Intravenosa con Dispositivos no Permanentes en Adultos. Guía de Práctica Clínica sobre Terapia Intravenosa con Dispositivos no Permanentes en Adultos [monografía en Internet]. Madrid, España: Ministerio de Sanidad, Servicios Sociales e Igualdad y Agencia de Evaluación de Tecnologías Sanitarias de Andalucía (AETSA); 2014 [consultada 22 nov 2018]. Disponible en: https://bit.ly/2eHWc3x

2. Grupo de trabajo de la Guía de Práctica Clínica sobre lactancia materna. Guía de Práctica Clínica sobre lactancia materna [monografía en Internet]. Madrid, España: Ministerio de Sanidad, Servicios Sociales e Igualdad y Agencia de Evaluación de Tecnologías Sanitarias del País Vasco-OSTEBA; 2017 [consultada 22 nov 2018]. Disponible en: https://bit.ly/2srihx1

3. International Organization for Standardization (ISO). ISO 5807:1985 Information processing Documentation symbols and conventions for data, program and system flowcharts, program network charts and system resources charts [monografía en Internet]. Geneva, Switzerland: ISO; 2005 [consultada 22 nov 2018]. Disponible en: https://www.iso.org/standard/11955.html

4. Grupo de trabajo de la Guía de Práctica Clínica sobre el Manejo de la Depresión en el Adulto. Guía de Práctica Clínica sobre el Manejo de la Depresión en el Adulto [monografía en Internet]. Madrid, España: Ministerio de Sanidad, Servicios Sociales e Igualdad y Agencia de Evaluación de Tecnologías Sanitarias de Galicia (avalia-t); 2014. [consultada 22 nov 2018]. Disponible en: https://bit.ly/1VgMS86

5. Rumbaugh J, Jacobson I, Booch G. The unified modeling language reference manual. $2^{\circ}$ edition. Reading, Massachusetts, USA: Pearson Higher Education; 2004.

6. Object Management Group (OMG). Business Process Model and Notation (BPMN) version 2.0.2 [monografía en Internet]. Needham, Massachusetts, USA: OMG; 2011 [consultada 22 nov 2018]. Disponible en: https://bit.ly/2Brmkg6

7. Van Der Aalst WM, Ter Hofstede AH. YAWL: yet another workflow language. Inf Syst. 2005;30(4):245-75. DOI: 10.1016/j.is.2004.02.002

8. Peterson JL. Petri net theory and the modeling of systems. Englewood Cliffs, New Jersey, USA: Prentice-Hall International; 1981. 
9. Braun R, Burwitz M, Schlieter H, Benedict M. Clinical processes from various angles - amplifying BPMN for integrated hospital management. In: Institute of Electrical and Electronics Engineers (IEEE) International Conference on Bioinformatics and Biomedicine (BIBM), 2015 nov 9-12. Washington, DC, USA: IEEE; 2015. DOI: 10.1109/BIBM.2015.7359794

10. Braun R, Schlieter H, Burwitz M, Esswein W. BPMN4CP Revised - Extending BPMN for Multiperspective Modeling of Clinical Pathways. In: 49th Hawaii International Conference on System Sciences (HICSS), 2016 jan 5-8. Koloa, Hawaii, USA: Institute of Electrical and Electronics Engineers (IEEE); 2016. DOI: 10.1109/HICSS.2016.407

11. Strasser M, Pfeifer F, Helm E, Schuler A, Altmann J. Defining and reconstructing clinical processes based on IHE and BPMN 2.0. In: Moen A, Andersen SK, Aarts J, Hurlen P (editors). User Centred Networked Health Care - Studies in health technology and informatics. Amsterdam, The Netherlands: IOS Press; 2011. pp 482-86. DOI: 10.3233/978-1-60750-806-9-482

12. Ramos-Merino M, Santos-Gago JM, Álvarez-Sabucedo LM, Sanz-Valero J. BPMN-E2: a BPMN extension for an enhanced workflow description. Softw Syst Model. 2018. DOI: 10.1007/s10270018-0669-2

13. Merino MR, Sabucedo LMÁ, Gago JMS, Rorís VMA. A BPMN Extension for the Annotation of HACCP Plans in Hospital Protocols. In: 6th World Conference on Information Systems and Technologies (WorldCIST): Trends and Advances in Information Systems and Technologies. 2018 mar 27-29. Naples, Italy: Springer; 2018. pp. 606-15. DOI: 10.1007/978-3-319-77703-0_60

14. Ramos-Merino M, Álvarez-Sabucedo LM, Santos-Gago JM, Sanz-Valero J. A BPMN Based Notation for the Representation of Workflows in Hospital Protocols. J Med Syst. 2018;42(10):e181. DOI: 10.1007/s10916-018-1034-2.

15. Mortimore S, Wallace C. An introduction to HACCP. In: Mortimore S, Wallace C. HACCP A Practical Approach. $2^{\circ}$ edition. Boston, Massachusetts, USA: Springer; 1998. pp. 1-11. DOI: 10.1007/978-1-4615-5781-4_1

16. Bernabeu-Martínez MA, Ramos Merino M, Santos Gago JM, Álvarez Sabucedo LM, WandenBerghe C, Sanz-Valero J. Guidelines for safe handling of hazardous drugs: A systematic review. PLoS One. 2018;13(5):e0197172. DOI: 10.1371/journal.pone.0197172.

17. Hand DJ. Principles of data mining. Drug Saf. 2007;30(7):621-2. DOI: 10.2165/00002018200730070-00010

18. Walker SJ. Big data: A revolution that will transform how we live, work, and think. Int $J$ Advert. 2014;33(1):181-3. DOI: 10.2501/IJA-33-1-181-183 